\title{
Detection of Neospora caninum Tachyzoites in Canine Cerebrospinal Fluid
}

\author{
Lluís Gaitero, Sònia Añor, Patrícia Montoliu, Ángeles Zamora, and Martí Pumarola
}

\begin{abstract}
A 4-year-old female Labrador Retriever was referred to the Veterinary Teaching Hospital of the Autonomous University of Barcelona because of progressive, generalized ataxia and depressed mental status of 5 days' duration. Before the onset of signs of neurologic dysfunction, the dog had been anorexic, lethargic, and hyperthermic for 7-10 days. The dog had been treated with doxycycline $(5 \mathrm{mg} / \mathrm{kg}$ of body weight PO q12h) by the referring veterinarian without clinical improvement. During that time, general attitude, condition, and gait had progressively worsened. Over the 12 hours before presentation, the dog experienced a generalized seizure. Routine vaccinations were current, the owners did not report any previous medical history, and the possibility of exposure to toxins was considered unlikely.

At presentation, abnormalities were not detected on physical examination. Neurologic examination identified stuporous mental status, intermittent opisthotonus with increased extensor tone in all 4 limbs (ie, decerebrate rigidity), and nonambulatory tetraparesis. Abnormalities on cranial nerve examination included absent oculocephalic movements, absent bilateral menace response, anisocoria (left eye mydriasis), absent pupillary light reflexes, and slightly decreased rightsided facial sensation. Ophthalmic examination identified bilaterally swollen (raised) optic disks and peripapillary retinal edema and inflammation suggesting bilateral optic neuritis. Neuroanatomic lesion localization was considered diffuse or multifocal (forebrain and brainstem). The differential diagnosis included inflammatory central nervous system (CNS) diseases, multifocal neoplastic processes, and vascular diseases. The dog experienced a generalized tonic-clonic seizure in the examination room, and a bolus of diazepam ${ }^{\mathrm{a}}(0.5 \mathrm{mg} /$ $\mathrm{kg}$ ) was administered IV. Initial emergency treatment consisted of a mannitol ${ }^{\mathrm{b}}$ bolus ( $1 \mathrm{~g} / \mathrm{kg} \mathrm{IV}$ over $20 \mathrm{~min}$ ),
\end{abstract}

From Servei de Neurologia i Neurocirurgia (Gaitero, Añor, Montoliu), Departament de Medicina (Pumarola) $i$ Cirurgia Animals, Facultat de Veterinària, Universitat Autònoma de Barcelona, 08193 Bellaterra: and Medical Resonancia Magnética, SA (Zamora), Barcelona, SPAIN. Previously presented at the ESVN 17th Annual Symposium held in Glasgow, Scotland, September 2004.

Reprint requests: Sonia Añor, DVM, PhD, Departament de Medicina i Cirurgia Animals, Facultat de Veterinària, Universitat Autònoma de Barcelona, 08193 Bellaterra, Barcelona, SPAIN; e-mail: sonia.anor@uab.es.

Received May 30, 2005; Revised July 4, 2005; Accepted July 29, 2005.

Copyright $(0) 2006$ by the American College of Veterinary Internal Medicine

0891-6640/06/2002-0029/\$3.00/0 dexamethasone $^{\mathrm{c}} \quad(0.25 \mathrm{mg} / \mathrm{kg} \quad \mathrm{IV})$, oxygen therapy $(50 \mathrm{ml} / \mathrm{kg} / \mathrm{min}$ via nasal catheter), and fluid therapy with $0.9 \%$ saline solution ${ }^{\mathrm{d}}$ at the rate of $6 \mathrm{ml} / \mathrm{kg} / \mathrm{h}$. Twelve hours later, the neurologic status was moderately improved. Pupillary function returned partially, but the direct pupillary light reflex in the left eye and the consensual pupillary light reflex in the right eye were still absent. Oculocephalic movements were present, but vertical positional nystagmus and bilateral positional ventral strabismus appeared.

Fluid therapy with $0.9 \% \mathrm{NaCl}$ was continued at a maintenance rate, and was supplemented with potassium at a dosage of $20 \mathrm{mEq} / \mathrm{L}$. Dexamethasone treatment was continued $(0.25 \mathrm{mg} / \mathrm{kg}$ IV q24h), and ranitidine $e^{\mathrm{e}}$ was initiated $(2 \mathrm{mg} / \mathrm{kg} \mathrm{IV} \mathrm{q12h})$ to try to protect the gastrointestinal tract from the effects of glucocorticoid therapy.

Results of hematologic and serum biochemical analyses, urinalysis, thoracic radiography, and abdominal ultrasonography indicated mild, non-regenerative (normocytic, normochromic) anemia (hematocrit, 31\%; reference range, $37-55 \%$; RBC, $4.73 \times 10^{6} / \mu \mathrm{L}$; reference range, $5.5-8.5 \times 10^{6} / \mu \mathrm{L}$ ), and mild-to-moderate polyclonal hypergammaglobulinemia $(2.03 \mathrm{~g} / \mathrm{dL}$; reference range, $0.3-0.8 \mathrm{~g} / \mathrm{dL})$. The absolute reticulocyte count was $9,460 / \mu \mathrm{L}$ (reference range, $0-60,000 / \mu \mathrm{L}$ ).

Three days after admission, magnetic resonance imaging (MRI) of the brain and cranial cervical part of the spinal cord was performed with a 1.5-tesla superconducting magnet. ${ }^{\mathrm{f}}$ Diazepam $(0.5 \mathrm{mg} / \mathrm{kg}$ IV) was administered, followed by a constant-rate infusion of propofol ${ }^{\mathrm{g}}$ to maintain a light plain of anesthesia. Sagittal, transverse, and dorsal images were obtained with a Fast-spin echo (SE) T2-weighted sequence (TR 5000/TE 112) and a SET1-weighted sequence after IV administration of $0.1 \mathrm{mmol}$ of gadoteric $\mathrm{acid}^{\mathrm{h}} / \mathrm{kg}$. Images indicated increased leptomeningeal thickness, with diffuse rostro- and caudotentorial meningeal contrast enhancement (Fig 1) extending into the cranial cervical part of the spinal cord segment. The leptomeningeal thickening was more pronounced along the base of the cranium, especially at the diencephalic level, where a mild mass effect over the brainstem and temporal lobe was seen. In addition, a large, left cortical-subcortical parietotemporal lesion was observed. This second lesion was homogeneously hyperintense and well delineated on Fast SE T2-weighted images and was isointense on SE T1-weighted images. Perilesional edema or contrast enhancement was not evident, but a mass effect over the left lateral ventricular wall (Fig 2) was observed. All of these MRI findings were considered consistent with diffuse inflammatory 


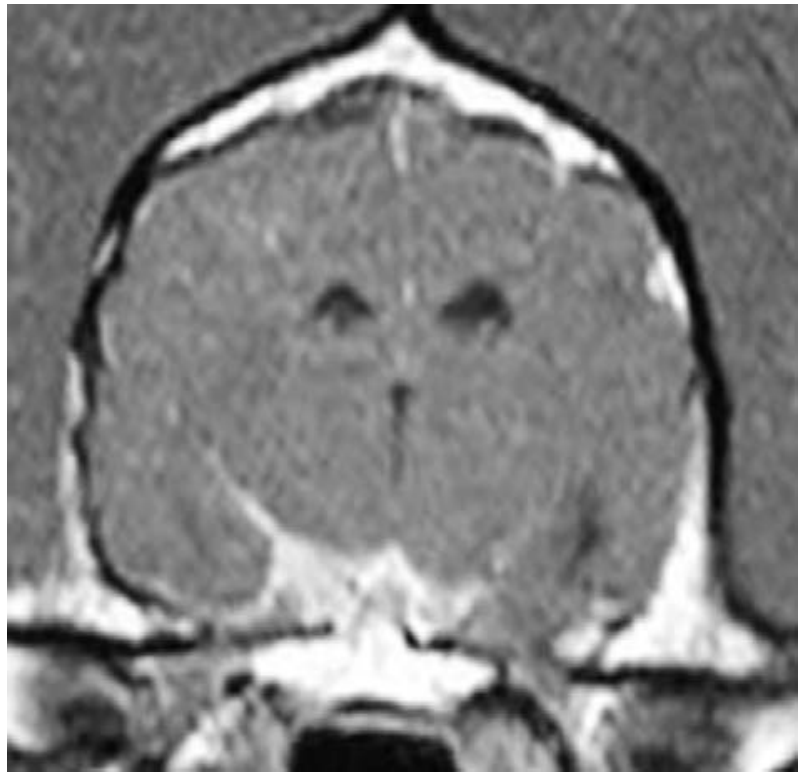

Fig 1. Transverse postcontrast T1-weighted magnetic resonance (MR) scan demonstrating leptomeningeal infiltration that extends to the cavernous sinus and produces mass effect over the temporal lobe and brainstem.

leptomeningeal infiltration, with a secondary left cortical-subcortical focal parenchymal ischemic lesion.

Immediately after the MRI procedure, cerebrospinal fluid (CSF) was collected from the cerebellomedullary cistern and was analyzed. Analysis of CSF revealed a severe, mixed, mostly mononuclear pleocytosis $(1,450$ $\mathrm{WBC} / \mu \mathrm{L}$; reference range, fewer than $5 \mathrm{WBC} / \mu \mathrm{L})$, and increased protein concentration $(992 \mathrm{mg} / \mathrm{dL}$; reference range, $<27 \mathrm{mg} / \mathrm{dL}$ ). ${ }^{1-3}$ Examination of smears of cytocentrifuged samples disclosed the presence of numerous protozoal tachyzoites (Fig 3). The organisms were small and bow-shaped. They were located intracellularly within inflammatory cells and as free extracellular tachyzoites (Fig 3). A protozoal CNS infection was diagnosed. Treatment with clindamycin ${ }^{i}$ $(11 \mathrm{mg} / \mathrm{kg}$ IV q12h) was instituted. Glucocorticoid treatment was stopped, and maintenance fluid therapy with $0.9 \%$ saline solution was continued.

The patient's status gradually deteriorated over time despite mild initial improvement. The neurologic condition progressed to comatose mental status, decerebrate posture, and bilateral mydriasis with absent bilateral pupillary light reflexes and oculocephalic movements. Death attributable to cardiorespiratory arrest occurred 5 days after admission.

Results of a complete postmortem examination did not indicate any macroscopic changes. The brain, spinal cord, and other extraneural tissues were fixed in buffered $10 \%$ formalin, embedded in paraffin, and sectioned. Tissue sections were stained with hematoxylin and eosin. Microscopic examination of the brain identified a severe, predominantly ventral, diffuse, mixed inflammatory infiltrate affecting the leptomeninges and extending into the adjacent nervous parenchyma (thalamus, optic chiasm, pituitary gland, rhinen-

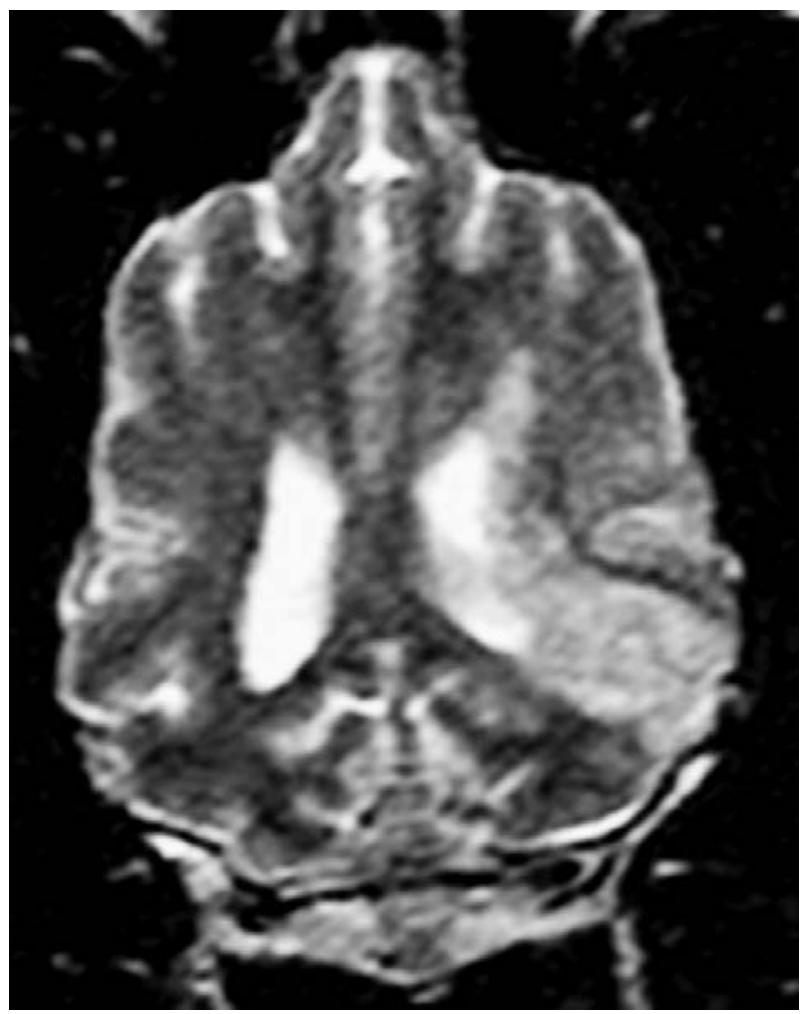

Fig 2. Dorsal, Fast-SE, T2-weighted MR scan showing a cortical-subcortical parenchymal lesion consistent with a secondary infarct.

cephalon, hippocampus). In the leptomeninges, the infiltrate consisted of plasma cells, lymphocytes, macrophages, and eosinophils. Gliosis, neuronal necrosis, and protozoal cysts were observed in the neuroparenchyma. Large areas of neuronal necrosis, gitter cells, proliferating vessels, white matter spongiosis, and small hemorrhages were present in the left temporal cortex. These inflammatory lesions also were observed, to a lesser extent, in the cranial cervical spinal cord segments. Lesions or protozoal organisms were not observed in other organs.

Immunohistochemical (IHC) analysis of selected deparaffinized brain sections with rabbit polyclonal antiNeospora caninum-specific antibody (provided by Dr J.P. Dubey) indicated the presence of $N$. caninumpositive cysts and free tachyzoites (Fig 4). Tachyzoites and tissue cysts did not stain with rabbit polyclonal antiToxoplasma gondii antibody (also provided by $\mathrm{Dr}$ Dubey). Multifocal meningoencephalomyelitis caused by $N$. caninum infection was the final diagnosis.

Neosporosis is an important cause of meningoencephalomyelitis, polymyositis, and polyradiculoneuritis in dogs throughout the world. Dogs serve as intermediate and definitive hosts of the apicomplexa protozoal parasite, $N$. caninum, because parasite oocysts have been found in canine feces. ${ }^{4-9}$ Tachyzoites and the tissue cyst-forming bradyzoites comprise the intermediate host parasite cycle stages. Tissue cysts are found principally in the CNS. Recently, tissue cysts also have been reported in the skeletal muscles of naturally infected 


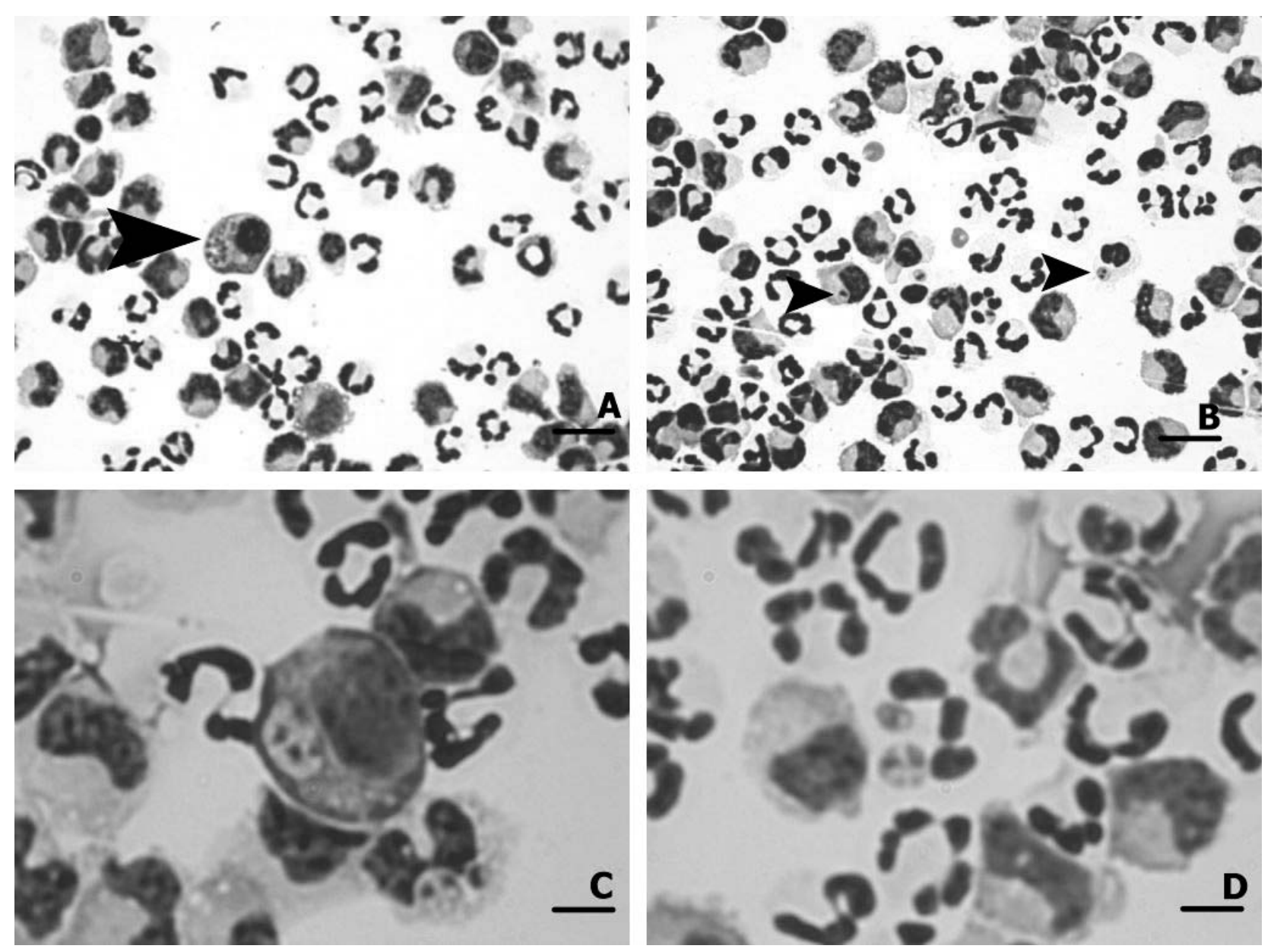

Fig 3. (A, B) Cerebrospinal fluid. Tachyzoites within macrophages (arrowheads). Quick panoptic. Bar $=25 \mu \mathrm{m}$. (C) Tachyzoites within a macrophage in the CSF. Quick panoptic. Bar $=100 \mu \mathrm{m}$. (D) Free extracellular and intraneutrophilic tachyzoites in the CSF. Quick panoptic. Bar $=10 \mu \mathrm{m}$.

dogs and cattle. ${ }^{10}$ Transplacental infection is believed to be the common route of transmission, but postnatal infection must occur to maintain infection at seroprevalence rates reported in canine populations. ${ }^{11}$ Dogs can also acquire the infection by ingestion of infected tissues. ${ }^{4,89}$ Most cases of clinical neosporosis are reported in dogs younger than 1 year.,.$^{5,6,12,13}$ Affected young dogs often develop rapidly progressive ascending paresis, usually with a characteristic rigid pelvic limb hyperextension caused by polymyositis, polyradiculoneuritis, and meningoencephalomyelitis. Puppies also may develop multiple organ involvement and sudden death attributable to myocardial infection. ${ }^{4,6,7,14}$ Adult dogs can be infected, but usually do not develop pelvic limb hyperextension. In adult animals, clinical signs of multifocal CNS involvement are more common, but polymyositis, dermatitis, or multiorgan infection also have been described. ${ }^{4,6,13,15}$

Detection of high serum $N$. caninum titer by means of an immunoglobulin indirect fluorescent antibody test indicates exposure to the organism and, in conjunction with compatible clinical signs of disease, neosporosis should be suspected. A positive diagnosis, however, requires identification of the parasite in CSF or tissues.
The parasite has been detected in CSF by polymerase chain reaction (PCR) analysis, ${ }^{13}$ in muscle biopsy specimens, ${ }^{5,16}$ lung aspirates, and cutaneous lesions. ${ }^{6,12}$ Recently, a multiplex PCR assay for detection of $N$. caninum in biological samples from clinically affected dogs has been developed. ${ }^{17}$ Other diagnostic tests that may support the diagnosis of neosporosis include determination of antibody titer in the $\mathrm{CSF},{ }^{12}$ presence of increased serum enzyme activities indicative of hepatic or muscular lesions, ${ }^{6,12}$ and presence of electromyographic abnormalities in skeletal muscles. ${ }^{14}$

To the authors' knowledge, this case report provides the first images of $N$. caninum tachyzoites in canine CSF and the MRI findings of meningoencephalomyelitis caused by $N$. caninum infection. Despite the number of cases and CSF specimens analyzed, cytologic detection of $N$. caninum is extremely rare. Due to the typical nonspecific CSF pattern in inflammatory CNS diseases, finding organisms in CSF cytologic smears becomes essential for diagnostic and therapeutic purposes. To the authors' knowledge, the only previously reported clinical case in which $N$. caninum tachyzoites were found in the CSF was one of a series in a retrospective study that evaluated signalment, history, laboratory data, and 

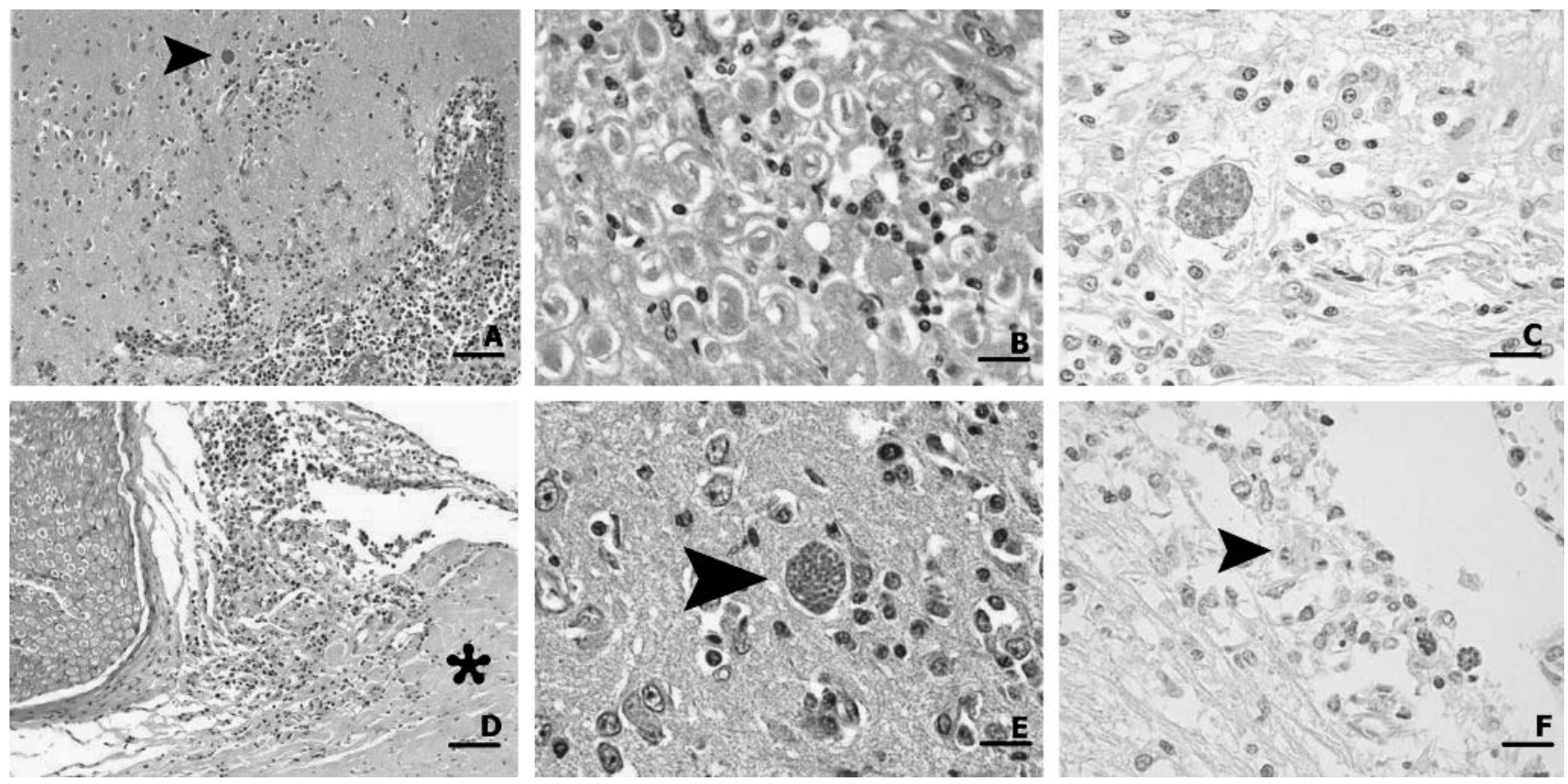

Fig 4. Photomicrographs of sections from parts of the brain of the Labrador Retriever of this report. (A) Ventral aspect of the diencephalon. Notice the marked inflammatory reaction and the protozoal cyst (arrowhead). Hematoxylin and eosin $(\mathrm{HE})$. Bar $=100 \mu \mathrm{m}$. (B) Trochlear nerve with mixed inflammatory infiltrate. HE. Bar $=25 \mu \mathrm{m}$. (C) Protozoal cyst of Neospora caninum with positive reaction to immunohistochemical (IHC) staining. Bar $=25 \mu \mathrm{m}$. (D) Meningeal mixed inflammatory infiltrate. *Dura mater. HE. Bar $=100 \mu \mathrm{m}$. (E) Mesencephalic gray matter. Notice protozoal cyst (arrowhead). HE. Bar $=25 \mu \mathrm{m}$. (F) Protozoal cyst and tachyzoites (arrowhead) with $N$. caninum-positive reaction to IHC analysis. Bar $=25 \mu \mathrm{m}$.

histologic sections from 23 dogs previously determined to have a toxoplasmosis-like illness. ${ }^{7}$ Toxoplasma-like organisms had been described in the CSF of a dog and later identified as $N$. caninum on the basis of histologic structural differences with Toxoplasma gondii and on the absence of reaction to anti- $T$. gondii serum in an immunoperoxidase test. In the dog of this report, antemortem diagnosis of neosporosis was based on direct observation of Neospora tachyzoites on CSF cytologic examination and was confirmed by specific IHC analysis for $N$. caninum in tissue sections.

In human beings, $T$. gondii organisms have been identified on cytologic examination of CSF. ${ }^{18-20}$ Toxoplasmosis is asymptomatic in $90 \%$ of immunocompetent human beings, but $15 \%$ to $46 \%$ of infected immunocompromised adult patients develop toxoplasmic encephalitis. ${ }^{18}$ However, direct identification of $T$. gondii in the CSF of human beings also is uncommon and only 5 cases have been documented to date. ${ }^{18-20}$ Four of these 5 patients were immunocompromised and died within days of diagnosis, similar to the outcome in the Labrador Retriever of this report. Whether the presence of Neospora tachyzoites in this dog was a result of an underlying immunodeficiency or was associated with a more virulent strain of $N$. caninum is unknown. Immunodeficiency could also have been caused or aggravated by administration of glucocorticoids before collection of CSF. Whether observation of the parasite in the CSF would have been possible if glucocorticoids had not been administered also is unknown and represents a possible explanation for the large numbers of parasites seen and the rapid progression of the disease.

Although the literature indicates that a breed predisposition has not been proven, most canine cases of neosporosis have been described in Labrador Retrievers, Boxers, Greyhounds, Golden Retrievers, and Basset Hounds. ${ }^{4,6}$ To date, primary immunodeficiencies in these breeds have only been reported in Basset Hounds. ${ }^{21}$

Magnetic resonance imaging (MRI) is useful for differentiating inflammatory diseases from neoplastic masses. ${ }^{22}$ In the dog of this report, MR images obtained were consistent with an inflammatory process and vascular complications. The MRI data confirmed the neuroanatomic localization of the lesions and provided information about the severity and extent of the infection in the CNS.

On the basis of the CSF findings in this dog, an antemortem diagnosis of CNS neosporosis in dogs can be made by identification of Neospora tachyzoites in CSF samples. Antemortem diagnosis of neosporosis is commonly made on the basis of clinical signs of disease, together with diagnostic serum antibody titer or detection of the parasite by PCR analysis. Often, a definitive diagnosis cannot be reached before postmortem histopathologic examination. This case report demonstrates that antemortem detection of N. caninum tachyzoites in CSF is feasible, and if findings are positive, is one of the simplest and quickest tests to obtain a definitive antemortem diagnosis. 


\section{Footnotes}

${ }^{\text {a }}$ Valium 10 ampollas, Roche Farma, SA, 28027 Madrid, Spain

${ }^{\mathrm{b}}$ Osmofundina, B. Braun Medical, SA, Rubí (Barcelona), Spain

${ }^{\mathrm{c}}$ Resdex Inyectable, Schering Plough Santé Animale, 49500 Segré, France

${ }^{\mathrm{d}}$ Fisiológico Braun, B. Braun Medical, SA, Rubí (Barcelona), Spain

${ }^{\mathrm{e}}$ Zantac Inyectable, GlaxoSmithKline, SA, 28760 Tres Cantos (Madrid), Spain

${ }^{\mathrm{f}}$ Toshiba MR system MRT-150A 1.5 Tesla, Toshiba Medical Systems, SA, 28108 Alcobendas (Madrid), Spain

${ }^{\mathrm{g}}$ Propofol-Lipuro 1\%, B. Braun Melsungen AG, D-34209 Melsungen, Germany

${ }^{\text {h }}$ Dotarem Acido gadotérico, Guerbet. BP. 50400, F-95943 Roissy CdG Cedes, France

${ }^{\mathrm{i}}$ Dalacin Fosfato $600 \mathrm{mg}$ solución inyectable, Pharmacia Spain, SA, 08190 Sant Cugat Del Valles (Barcelona), Spain

\section{Acknowledgment}

The authors would like to thank Dr J.P. Dubey, United States Department of Agriculture, Agricultural Research Service, Animal and Natural Resource Institute, Animal Parasitic Diseases Laboratory, Beltsville, MD 20705-2350, USA, for providing the antiserum against Neospora caninum and Toxoplasma gondii.

\section{References}

1. Ducoté JM, Dewey CW. Neurodiagnostics. In: Dewey CW, ed. A Practical Guide to Canine and Feline Neurology. Ames, IA: Iowa State University Press; 2003:57-98.

2. Munana KR. Encephalitis and meningitis. Vet Clin North Am 1996;26:857-874.

3. Chrisman CL. Cerebrospinal fluid analysis. Vet Clin North Am 1992;22:781-810.

4. Dubey JP. Review of Neospora caninum and neosporosis in animals. Korean J Parasitol 2003;41:1-16.

5. Dubey JP. Recent advances in Neospora and neosporosis. Vet Parasitol 1999;84:349-367.

6. Dubey JP, Lindsay DS. A review of Neospora caninum and neosporosis. Vet Parasitol 1996;67:1-59.

7. Dubey JP, Carpenter JL, Speer CA, et al. Newly recognized fatal protozoan disease of dogs. J Am Vet Med Assoc 1988;192: 1269-1285.
8. McAllister MM, Dubey JP, Lindsay DS, et al. Dogs are definitive hosts of Neospora caninum. Int J Parasitol 1998;28: 1473-1478.

9. Lindsay DS, Dubey JP, Duncan RB. Confirmation that the dog is a definitive host for Neospora caninum. Vet Parasitol 1999;82:327-333.

10. Peters M, Lutkefels E, Heckeroth AR, Schares G. Immunohistochemical and ultrastructural evidence for Neospora caninum tissue cysts in skeletal muscles of naturally infected dogs and cattle. Int J Parasitol 2001;31:1144-1148.

11. Barber JS, Trees AJ. Naturally occurring vertical transmission of Neospora caninum in dogs. Int J Parasitol 1998; 28:57-64.

12. Ruehlmann D, Podell M, Oglesbee M, Dubey JP. Canine neosporosis: a case report and literature review. J Am Anim Hosp Assoc 1995;31:174-183.

13. Peters M, Wagner F, Schares G. Canine neosporosis: clinical and pathological findings and first isolation of Neospora caninum in Germany. Parasitol Res 2000;86:1-7.

14. Mayhew IG, Smith KC, Dubey JP, et al. Treatment of encephalomyelitis due to Neospora caninum in a litter of puppies. J Small Anim Pract 1991;32:609-612.

15. Ordeix L, Lloret A, Fondevila D, et al. Cutaneous neosporosis during treatment of pemphigus foliaceus in a dog. J Am Anim Hosp Assoc 2002;38:415-419.

16. Dubey JP, Dorough KR, Jenkins MC, et al. Canine neosporosis: clinical signs, diagnosis, treatment and isolation of Neospora caninum in mice and cell culture. Int J Parasitol 1998;28: 1293-1304.

17. Schatzberg SJ, Haley NJ, Barr SC, et al. Use of a multiplex polymerase chain reaction assay in the antemortem diagnosis of toxoplasmosis and neosporosis in the central nervous system of cats and dogs. Am J Vet Res 2003;64:1507-1513.

18. Brogi E, Cibas ES. Cytologic detection of Toxoplasma gondii tachyzoites in cerebrospinal fluid. Am $\mathrm{J}$ Clin Pathol 2000;114:951-955.

19. DeMent SH, Cox MC, Gupta PK. Diagnosis of central nervous system Toxoplasma gondii from the cerebrospinal fluid in a patient with acquired immunodeficiency syndrome. Diagn Cytopathol 1987;3:148-151.

20. Eggers C, Gross U, Klinker H, et al. Limited value of cerebrospinal fluid for direct detection of Toxoplasma gondii in toxoplasmic encephalitis associated with AIDS. J Neurol 1995;242: 644-649.

21. Perryman LE. Molecular pathology of severe combined immunodeficiency in mice, horses, and dogs. Vet Pathol 2004;41: 95-100.

22. Stewart WA, Parent JM-L, Towner RA, Dobson H. The use of magnetic resonance imaging in the diagnosis of neurological disease. Can Vet J 1992;33:585-590. 